\title{
Editorial: The Immunomodulatory Roles of Adipocytes
}

\author{
David Bradley ${ }^{1 *}$, Aimin $\mathrm{Xu}^{2,3}$ and Willa A. Hsueh ${ }^{1 *}$ \\ ${ }^{1}$ Diabetes and Metabolism Research Center, Division of Endocrinology, Diabetes \& Metabolism, Department of Internal \\ Medicine, The Ohio State University Wexner Medical Center, Columbus, OH, United States, ${ }^{2}$ State Key Laboratory of \\ Pharmaceutical Biotechnology, The University of Hong Kong, Hong Kong, Hong Kong SAR, China, ${ }^{3}$ Department of \\ Medicine, The University of Hong Kong, Hong Kong, Hong Kong SAR, China
}

Keywords: adipocyte, innate and adaptive immune response, exosomes, adipokine cytokines, metabolic disease

\section{Editorial on the Research Topic}

\section{The Immunomodulatory Roles of Adipocytes}

Obesity is a global epidemic (1) associated with a state of low-grade, chronic inflammation that enhances the risk of numerous complications, including type 2 diabetes (T2D), non-alcoholic fatty liver disease (NAFLD) and cirrhosis, cardiovascular disease (CVD), cancer, and Alzheimer's Disease, among others (2-7). A major driver of these conditions is the profound inflammatory changes that occur within the adipose tissue (AT) microenvironment, at the heart of which is the adipocyte. Our understanding of the role of the adipocyte in initiating and propagating innate

\section{OPEN ACCESS}

Edited and reviewed by: Pietro Ghezzi,

Brighton and Sussex Medical School, United Kingdom

*Correspondence: Willa A. Hsueh

Willa.Hsueh@osumc.edu David Bradley

Dbradley3@pennstatehealth.psu.edu

Specialty section:

This article was submitted to Inflammation,

a section of the journal

Frontiers in Immunology

Received: 01 December 2021 Accepted: 10 December 2021 Published: 23 December 2021

Citation:

Bradley D, Xu A and Hsueh WA (2021) Editorial:

The Immunomodulatory

Roles of Adipocytes.

Front. Immunol. 12:827281. doi: 10.3389/fimmu.2021.827281
(Blaszczak et al.) and adaptive (Song and Deng) immune responses in lean and obese states has expanded beyond its classical role in energy storage. The adipocyte produces over 600 cytokines and hormones, collectively called adipokines that modulate chronic inflammation, secretes extracellular matrix proteins that impact metabolism $(8,9)$, and serves as an immunomodulatory and antigen presenting cell to activate or suppress immune responses within AT and systemically (10). Therefore, the current Research Series "The Immunomodulatory Roles of the Adipocyte" highlights a wide range of critical factors originating from the adipocyte that mediate immunity and the metabolic syndrome including extracellular vesicle crosstalk (Huang and Xu), lipid metabolites (Park et al.) and ceramides (Chaurasia et al.), adipocyte fatty acid-binding protein (A-FABP) (Lee et al.), TANK-binding kinase 1 (TBK1) (Zhao and Saltiel), the oncostatin M (Sanchez-Infantes and Stephens), clusterin (Wittwer and Bradley), and leptin (Kiernan and MacIver), illustrating the multi-faceted role of the adipocyte (Figure 1). Taken together, this series underscores the much underappreciated role of the adipocyte in the instigation and perpetuation of local and systemic inflammation, leading to the multiple inflammatory-induced complications of obesity.

An increasingly recognized means of cell-cell communication is through extracellular vesicles (EVs). Huang and Xu nicely summarize the mechanisms by which AT extracellular vesicles (exosomes, microvesicles, and apoptotic bodies) mediate intercellular communications and interorgan crosstalk, particularly focusing on adipocyte-derived EVs (ADEVs). Exosomes (30-100nm in diameter) arise from multivesicular bodies and are either degraded by the lysosomal pathway or fuse with the plasma membrane and are released from the cell, while microvescicles (MVs, 100-1000 nm is diameter) are pinched off from the plasma membrane and released. EV cargo consists of microRNAs (miRs), mRNAs, proteins, and lipids and are taken up by cells to influence cell development, metabolism, function, and other activities. ADEV production is markedly increased in human and mouse obesity (11). ADEVs impact local immune cells and have been shown to 


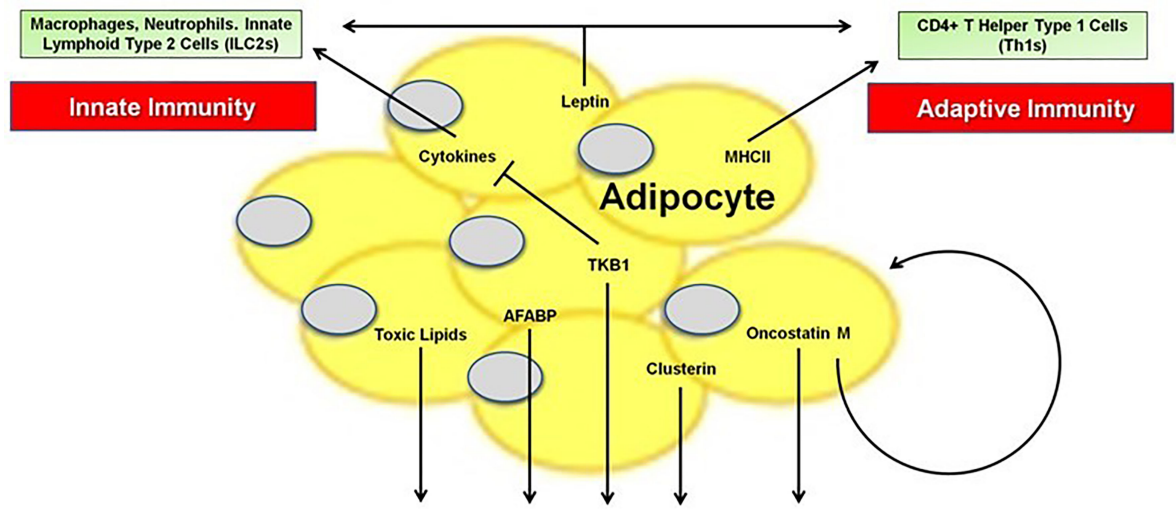

Insulin Resistance and Metabolic Syndrome

FIGURE 1 | The Innate and Adaptive Immune Functions of the Adipocyte.

activate AT macrophages (11) and promote monocyte to macrophage conversion (12-14). Adipocytes have been suggested to contribute substantially to miRs in circulating EVs, since adipocyte-specific loss of miR production resulted in a 4-fold drop in EV miR cargo (15). However, it remains unclear how many ADEVs enter the bloodstream (11). Figure 2 of the Huang and Xu review illustrates how ADEVs and their cargo act upon distal organs (including liver, skeletal muscle, pancreas, and brain) to influence the immune system as well as systemic metabolism, while Table 1 summarizes specific functions of various cargo. Finally, this review suggests modified EVs can be used as therapy for metabolic and other diseases.

Activation of a pro-inflammatory pathway leads to the secretion of numerous cytokines $(16,17)$ that enhance adipocyte lipolysis (18-20), leading to toxic fatty acid species (Chaurasia et al.) and impaired insulin sensitivity $(21,22)$. Chaurasia et al. review the wide-ranging effects of adipocytederived lipotoxicity on inflammation and peripheral tissue dysfunction. Specifically, ceramides, which are sphingolipids located in the cell membrane and within the cell cytosol, associate not only with pro-inflammatory cytokines and circulating free fatty acids, but many obesity-related conditions including insulin resistance, T2D, NAFLD, chronic kidney disease, and adverse CV events including mortality. Inhibition of ceramide synthesis specifically within the adipocyte improves insulin resistance and several of these metabolic derangements in mice, underscoring the role of the adipocyte in providing toxic lipids to incite inflammation (23). Inhibiting ceramide synthesis may be a useful therapeutic strategy for metabolic syndrome. Park et al. further discuss an integrated view of how adipocytes communicate with adipose immune cells using lipid metabolites. Invariant natural killer T (iNKT) cells and $\gamma / \delta$ T cells, rapidly respond to changes in lipid metabolism through sensing lipid antigens loaded on antigen presenting cells (APCs). iNKT cells secrete IL-2, IL-4 and IL-10 which support immunosuppressive regulatory $\mathrm{T}$ cells (Tregs), while IL-4 and IL-10 promote antiinflammatory macrophage M2 polarization (24). However, the lipid antigen is unknown and whether lipid-activated iNKT cells are anti- or proinflammatory remains controversial $(25,26)$. Similarly, $\gamma / \delta$ T cells are abundantly present in AT and actively interact with adipocytes, but their role in inflammation is also unclear. Nevertheless, iNKT cells and $\gamma / \delta$ T cells are models by which adipocytes can present a lipid antigen to activate an immune cell.

Song and Deng further define the adipocyte as a novel APC, substantially contributing to adaptive immunity in AT. The adipocyte major histocompatibility II (MHCII) pathway is markedly enhanced in obesity during which it is stimulated primarily by interferon- $\gamma(\operatorname{IFN} \gamma)(10,27)$. Adipocyte antigen presentation to naïve $\mathrm{T}$ cells promotes inflammatory Th1 effector cell activation, while further production of IFN $\gamma$ fosters more adipocyte MHCII production, resulting in an escalating cycle of AT inflammation. Mice with genetic depletion of adipocyte MHCII, gain the same amount of weight as control mice, but are protected from AT inflammation and insulin resistance. Within obese AT, adipocytes also activate innate immune cells including macrophages and neutrophils to promote inflammation, while innate lymphoid cells type 2 may be metabolically protective, as reviewed by Blaszczak et al. They highlight the central role of the adipocyte in linking the innate and adaptive immune systems through the secretion of adipokines and cytokines; exosome release of lipids, hormones, and microRNAs; and contact interaction with other immune cells. During diet-induced obesity, a negative feedback loop involving the non-canonical IKK family member TBK1 regulates both innate immunity and glucose and energy metabolism within the adipocyte, as reviewed by Zhao and Saltiel. Upon activation by inflammatory cytokines and lipids, TBK1 suppresses NFKB signaling and attenuates AMP kinasemediated metabolic activity. They suggest the potential of a TBK1/IKK inhibitor as a new therapy for metabolic diseases. 
Adipocytes secrete a multitude of factors that have either proor anti-inflammatory functions that impact systemic metabolism. Leptin, one of the most well-known hormones secreted by adipocytes in obesity, in addition to its metabolic function, has important pro-inflammatory actions as comprehensively summarized by Kiernan and MacIver. They provide data suggesting that nearly every immune cell is activated by leptin. Oncostatin M (OSM) is a proinflammatory cytokine, elevated in human obesity and metabolic disease, which inhibits preadipocyte differentiation and enhances the proinflammatory response of adipocytes in a paracrine manner (Sanchez-Infantes and Stephens). However, loss of this system by genetic ablation of the OSM receptor in adipocytes, in contrast to these findings, also aggravates glucose homeostasis, so Sanchez-Infantes and Stephens argue that some adipocyte inflammation is necessary for normal metabolic function. Lee et al. focus on A-FABP, a lipid chaperone abundantly secreted from adipocytes and macrophages, as a key player mediating adipose-vascular cross-talk. A-FABP, in part via its activation of c-Jun $\mathrm{NH} 2$-terminal kinase (JNK) and activator protein-1 (AP-1), forms a positive feedback loop to perpetuate inflammatory responses. In mice, selective JNK inactivation in the AT significantly reduced expression of A-FABP and circulating AFABP levels and alleviated high fat high cholesterol diet-induced atherosclerosis (28). In humans, raised circulating AFABP levels are associated with incident metabolic syndrome, T2D and CVD, as well as nonalcoholic steatohepatitis, diabetic nephropathy and adverse renal outcomes, all conditions closely related to inflammation and enhanced CV mortality (Lee et al.; 29-34). They suggest that A-FABP may be a therapeutic target in obesityrelated complications. Finally, various extracellular matrix proteins (ECM) are secreted by adipocytes, which in turn, determines the AT architecture, enhances inflammation, and regulates systemic metabolism. As discussed by Wittwer and

\section{REFERENCES}

1. World Health Organization. Available at: https://www.who.int/news-room/ fact-sheets/detail/obesity-and-overweight.

2. Weisberg SP, McCann D, Desai M, Rosenbaum M, Leibel RL, Ferrante AW Jr. Obesity is Associated With Macrophage Accumulation in Adipose Tissue. J Clin Invest (2003) 112:1796-808. doi: 10.1172/JCI200319246

3. Schwartz MA, Schaller MD, Ginsberg MH. Integrins: Emerging Paradigms of Signal Transduction. Annu Rev Cell Dev Biol (1995) 11:549-99. doi: 10.1146/ annurev.cb.11.110195.003001

4. Akhtar DH, Iqbal U, Vazquez-Montesino LM, Dennis BB, Ahmed A. Pathogenesis of Insulin Resistance and Atherogenic Dyslipidemia in Nonalcoholic Fatty Liver Disease. J Clin Trans Hepatol (2019) 7:362-70. doi: 10.14218/JCTH.2019.00028

5. Kiliaan AJ, Arnoldussen IA, Gustafson DR. Adipokines: A Link Between Obesity and Dementia? The Lancet. Neurology (2014) 13:913-23. doi: 10.1016/S1474-4422(14)70085-7

6. Powell-Wiley TM, Poirier P, Burke LE, Despres JP, Gordon-Larsen P, Lavie CJ, et al. Obesity and Cardiovascular Disease: A Scientific Statement From the American Heart Association. Circulation (2021) 143:e984-1010. doi: 10.1161/ CIR.0000000000000973

7. Deng T, Lyon CJ, Bergin S, Caligiuri MA, Hsueh WA. Obesity, Inflammation, and Cancer. Annu Rev Pathol (2016) 11:421-49. doi: 10.1146/annurevpathol-012615-044359
Bradley (8), adipocyte ECM production is amplified in obesity, resulting in AT fibrosis and adipocyte hypoxia. Clusterin (apolipoprotein J), an ECM-related protein whose expression and secretion in adipocytes is higher in human obesity, is associated with multiple metabolic syndrome components and $\mathrm{CV}$ risk and has key effects centrally to modulate amyloid-beta in Alzheimer's Disease. The insulin antagonizing effects of clusterin appear to be in the liver (8).

In summary, the adipocyte exerts immunomodulatory functions via multiple novel mechanisms to regulate inflammation and contribute to obesity-related disease. The original research articles and review papers included in this issue present a range of topics under active investigation. Understanding this function and how it impacts other AT immune cells and obesity-related complications is critical to prevention and treatment. Yet, despite a recognition of the importance of adipocytes in inflammatory dysregulation, the mechanisms underlying the inflammatory regulation of these disorders are not fully understood and should remain a critical focus for future investigation.

\section{AUTHOR CONTRIBUTIONS}

All authors listed have made a substantial, direct and intellectual contribution to the work, and approved it for publication.

\section{FUNDING}

This study was supported by grants from the American Diabetes Association 1-16-ICTS-049, The National Institutes of Health KL2 Scholar Award KL2TR001068 and HL135622.

8. Bradley D, Blaszczak A, Yin Z, Liu J, Joseph JJ, Wright V, et al. Clusterin Impairs Hepatic Insulin Sensitivity and Adipocyte Clusterin Associates With Cardiometabolic Risk. Diabetes Care (2019) 42:466-75. doi: 10.2337/dc180870

9. Crewe C, An YA, Scherer PE. The Ominous Triad of Adipose Tissue Dysfunction: Inflammation, Fibrosis, and Impaired Angiogenesis. J Clin Invest (2017) 127:74-82. doi: 10.1172/JCI88883

10. Deng T, Lyon CJ, Minze LJ, Lin J, Zou J, Liu JZ, et al. Class II Major Histocompatibility Complex Plays an Essential Role in Obesity-Induced Adipose Inflammation. Cell Metab (2013) 17:411-22. doi: 10.1016/j.cmet. 2013.02.009

11. Flaherty SE, Grijalva 3A, Xu X, Ables E, Nomani A, Ferrante AWJr. A LipaseIndependent Pathway of Lipid Release and Immune Modulation by Adipocytes. Science (2019) 363:989-93. doi: 10.1126/science.aaw2586

12. Deng ZB, Poliakov A, Hardy RW, Clements R, Liu C, Liu Y, et al. Adipose Tissue Exosome-Like Vesicles Mediate Activation of Macrophage-Induced Insulin Resistance. Diabetes (2009) 58:2498-505. doi: 10.2337/db09-0216

13. Zhang Y, Mei H, Chang X, Chen F, Zhu Y, Han X. Adipocyte-Derived Microvesicles From Obese Mice Induce M1 Macrophage Phenotype Through Secreted Mir-155. J Mol Cell Biol (2016) 8:505-17. doi: 10.1093/jmcb/mjw040

14. Liu Z, Gan L, Zhang T, Ren Q, Sun C. Melatonin Alleviates Adipose Inflammation Through Elevating Alpha-Ketoglutarate and Diverting Adipose-Derived Exosomes to Macrophages in Mice. J Pineal Res (2018) 64:e12455. doi: 10.1111/jpi.12455 
15. Thomou T, Mori MA, Dreyfuss JM, Konishi M, Sakaguchi M, Wolfrum C, et al. Adipose-Derived Circulating Mirnas Regulate Gene Expression in Other Tissues. Nature (2017) 542:450-5. doi: 10.1038/nature21365

16. Olefsky JM, Glass CK. Macrophages, Inflammation, and Insulin Resistance. Annu Rev Physiol (2010) 72:219-46. doi: 10.1146/annurev-physiol-021909-135846

17. Agwunobi AO, Reid C, Maycock P, Little RA, Carlson GL. Insulin Resistance and Substrate Utilization in Human Endotoxemia. J Clin Endocrinol Metab (2000) 85:3770-8. doi: 10.1210/jcem.85.10.6914

18. Holland WL, Bikman BT, Wang LP, Yuguang G, Sargent KM, Bulchand S, et al. Lipid-Induced Insulin Resistance Mediated by the Proinflammatory Receptor TLR4 Requires Saturated Fatty Acid-Induced Ceramide Biosynthesis in Mice. J Clin Invest (2011) 121:1858-70. doi: 10.1172/JCI43378

19. Shulman GI. Cellular Mechanisms of Insulin Resistance. J Clin Invest (2000) 106:171-6. doi: 10.1172/JCI10583

20. Boden G. Fatty Acid-Induced Inflammation and Insulin Resistance in Skeletal Muscle and Liver. Curr Diabetes Rep (2006) 6:177-81. doi: 10.1007/s11892006-0031-x

21. Kelley DE, Mokan M, Simoneau JA, Mandarino LJ. Interaction Between Glucose and Free Fatty Acid Metabolism in Human Skeletal Muscle. J Clin Invest (1993) 92:91-8. doi: 10.1172/JCI116603

22. Ferrannini E, Barrett EJ, Bevilacqua S, DeFronzo RA. Effect of Fatty Acids on Glucose Production and Utilization in Man. J Clin Invest (1983) 72:1737-47. doi: 10.1172/JCI111133

23. Chaurasia B, Tippetts TS, Mayoral Monibas R, Liu J, Li Y, Wang L, et al. Targeting a Ceramide Double Bond Improves Insulin Resistance and Hepatic Steatosis. Science (2019) 365:386-92. doi: 10.1126/science.aav3722

24. Chawla A, Nguyen KD, Goh YP. Macrophage-Mediated Inflammation in Metabolic Disease. Nat Rev Immunol (2011) 11:738-49. doi: 10.1038/nri3071

25. Satoh M, Hoshino M, Fujita K, Iizuka M, Fujii S, Clingan CS, et al. AdipocyteSpecific CD1d-Deficiency Mitigates Diet-Induced Obesity and Insulin Resistance in Mice. Sci Rep (2016) 6:28473. doi: 10.1038/srep28473

26. Huh JY, Park J, Kim JI, Park YJ, Lee YK, Kim JB. Deletion of CD1d in Adipocytes Aggravates Adipose Tissue Inflammation and Insulin Resistance in Obesity. Diabetes (2017) 66:835-47. doi: 10.2337/db16-1122

27. Deng T, Liu J, Deng Y, Minze L, Xiao X, Wright V, et al. Adipocyte Adaptive Immunity Mediates Diet-Induced Adipose Inflammation and Insulin Resistance by Decreasing Adipose Treg Cells. Nat Commun (2017) 8:15725. doi: $10.1038 /$ ncomms15725

28. Kwok KHM, Cheng KKY, Hoo RLC, Ye D, Xu A, Lam KSL. Adipose-Specific Inactivation of JNK Alleviates Atherosclerosis in Apoe-Deficient Mice. Clin Sci (2016) 130:2087-100. doi: 10.1042/CS20160465
29. Chow WS, Tso AW, Xu A, Yuen MM, Fong CH, Lam TH, et al. Elevated Circulating Adipocyte-Fatty Acid Binding Protein Levels Predict Incident Cardiovascular Events in a Community-Based Cohort: A 12-Year Prospective Study. J Am Heart Assoc (2013) 2:e004176. doi: 10.1161/JAHA. 112.004176

30. Miyoshi T, Onoue G, Hirohata A, Hirohata S, Usui S, Hina K, et al. Serum Adipocyte Fatty Acid-Binding Protein is Independently Associated With Coronary Atherosclerotic Burden Measured by Intravascular Ultrasound. Atherosclerosis (2010) 211:164-9. doi: 10.1016/j.atherosclerosis.2010. 01.032

31. Tso AW, Lam TK, Xu A, Yiu KH, Tse HF, Li LS, et al. Serum Adipocyte Fatty Acid-Binding Protein Associated With Ischemic Stroke and Early Death. Neurology (2011) 76:1968-75. doi: 10.1212/WNL.0b013e31821e54b3

32. Francque SM, van der Graaff D, Kwanten WJ. Non-Alcoholic Fatty Liver Disease and Cardiovascular Risk: Pathophysiological Mechanisms and Implications. J Hepatol (2016) 65:425-43. doi: 10.1016/j.jhep.2016.04.005

33. Hoo RL, Lee IP, Zhou M, Wong JY, Hui X, Xu A, et al. Pharmacological Inhibition of Adipocyte Fatty Acid Binding Protein Alleviates Both Acute Liver Injury and Non-Alcoholic Steatohepatitis in Mice. J Hepatol (2013) 58:358-64. doi: 10.1016/j.jhep.2012.10.022

34. Lee CH, Cheung CYY, Woo YC, Lui DTW, Yuen MMA, Fong CHY, et al. Circulating Adipocyte Fatty Acid-Binding Protein Concentrations Predict Multiple Mortality Outcomes Among Men and Women With Diabetes. Clin Chem (2018) 64:1496-504. doi: 10.1373/clinchem.2018.289157

Conflict of Interest: The authors declare that the research was conducted in the absence of any commercial or financial relationships that could be construed as a potential conflict of interest.

Publisher's Note: All claims expressed in this article are solely those of the authors and do not necessarily represent those of their affiliated organizations, or those of the publisher, the editors and the reviewers. Any product that may be evaluated in this article, or claim that may be made by its manufacturer, is not guaranteed or endorsed by the publisher.

Copyright (c) 2021 Bradley, Xu and Hsueh. This is an open-access article distributed under the terms of the Creative Commons Attribution License (CC BY). The use, distribution or reproduction in other forums is permitted, provided the original author(s) and the copyright owner(s) are credited and that the original publication in this journal is cited, in accordance with accepted academic practice. No use, distribution or reproduction is permitted which does not comply with these terms. 